\title{
Test of CPT symmetry in cascade decays 1
}

\author{
Dong-Sheng Du, Zheng-Tao Wei \\ CCAST(World Laboratory), P.O.Box 8730, Beijing 100080, China \\ and \\ Institute of High Energy Physics, P.O.Box 918(4), Beijing 100039, China
}

\begin{abstract}
Cascade mixing provides an elegant place to study the $B^{0}-\bar{B}^{0}$ mixing. We use this idea to study the CPT violation caused by $B^{0}-\bar{B}^{0}$ mixing. An approximation method is adopted to treat the two complex $B^{0}-\bar{B}^{0}$ mixing parameters $\theta$ and $\phi$. A procedure to extract the parameters $\theta$ and $\phi$ is suggested. The feasibility of exploring the CPT violation and determining of $\theta$ and $\phi$ in the future B-factories and LHC-B is discussed.
\end{abstract}

PACS number: 11.30.-j, 13.25.-k.

\footnotetext{
${ }^{1}$ Supported in part by National Natural Science Foundation of China
} 


\section{Introduction}

The violations of the three discrete symmetries C, P, T have changed our ideas about the physical world. The discovery of CP violation in $K^{0}-\bar{K}^{0}$ complex was made long time ago. Recently, The direct $\mathrm{T}$ violation [1] and direct $\mathrm{CP}$ violation $\left(\operatorname{Re}\left(\epsilon^{\prime} / \epsilon\right) \neq 0\right)[2]$ are found experimentally. Only the combined CPT symmetry is left unbroken. The CPT theorem is the general result of the local, relativistic field theory. If it is violated, it will cause the fundamental crisis of our present particle theory. The recent tests of CPT violating effects give the bounds [3] $r_{K} \equiv 1$ $\frac{m_{\bar{K}^{0}}-m_{K^{0}}}{m_{K^{0}}} \mid \leq 10^{-18}$ and the very uncertain values $\operatorname{Re}(\delta)=\left(3.0 \pm 3.3_{\text {stat }} \pm 0.6_{\text {syst }}\right) \times 10^{-4}$, $\operatorname{Im}(\delta)=\left(-1.5 \pm 2.3_{\text {stat }} \pm 0.3_{\text {syst }}\right) \times 10^{-2}$. The tests of CPT symmetry in B system have been suggested from the theory [4] and phenomenology [5] [6]. In [5], the authors point out that CPT violation in $B^{0} \bar{B}^{0}$ mixing can lead to a dilepton asymmetry of neutral-B decays. They also discuss some general effects caused by CPT violation.

Neutral-meson interferometry is a powerful tool for investigating discrete symmetry. The $\mathrm{CP}$ violation $(\epsilon)$, direct $\mathrm{T}$ and direct $\mathrm{CP}$ violation are all observed in $K^{0} \bar{K}^{0}$ complex. In the decay chain $B \rightarrow J / \psi+K \rightarrow J / \psi+[f]$, neutral $\mathrm{K}$ mixing follows on the heels of $\mathrm{B}$ mixing. This mixing is called "cascade mixing" and the decay is "cascade decay". Cascade mixing has been got some theorists' interests [0] [8]. The extension to the decay chain $B \rightarrow D \rightarrow[f]_{D}$ for exploring new physics can be found in [9]. The advantage of cascade decay is that we can use the known $\mathrm{K}$ mixing parameter to determine the B mixing parameter. In [8], Kayser shows that the cascade decay contains more information than the usually discussed processes. So, cascade decay provides a complex and elegant window for the detail of the $B^{0}-\bar{B}^{0}$ mixing.

In this work, we shall make a detailed study of the CPT violating effects caused by $B^{0}-\bar{B}^{0}$ mixing in cascade decays. The $B^{0}-\overline{B^{0}}$ mixing is described by two complex phases $\theta, \phi$. We first take an approximation method to treat the parameters $\theta$ and $\phi$ and give the general formulas of the direct CPT and T asymmetry. Then we investigate how to extract the $B^{0}-\bar{B}^{0}$ mixing parameters. The feasibility of exploring the CPT violation in B-factories and LHC-B is discussed. Finally, we suggest a procedure to determine the two complex phases. 


\section{II. $B^{0}-\bar{B}^{0}$ mixing and CP, T, CPT Asymmetries}

Weak interaction can cause oscillation between $B^{0}$ and $\bar{B}^{0}$. The eigenstates of weak decays are not $B^{0}$ and $\bar{B}^{0}$ but their superpositions which have the simple exponential laws. The two eigenstates of $B^{0} \bar{B}^{0}$ mesons are given by

$$
\begin{aligned}
\mid B_{L}> & =\frac{1}{\sqrt{\left|p_{1}^{2}\right|+\left|q_{1}\right|^{2}}}\left[p_{1}\left|B^{0}>+q_{1}\right| \bar{B}^{0}>\right] \\
\mid B_{H}> & =\frac{1}{\sqrt{\left|p_{2}^{2}\right|+\left|q_{2}\right|^{2}}}\left[p_{2}\left|B^{0}>-q_{2}\right| \bar{B}^{0}>\right]
\end{aligned}
$$

and their eigenvalues are

$$
\begin{gathered}
\mu_{L}=m_{L}-\frac{i}{2} \Gamma_{L}=m_{B}-\frac{\Delta m_{B}}{2}-\frac{i}{2}\left(\Gamma_{B}+\frac{\Delta \Gamma_{B}}{2}\right)=m_{B}-\frac{i}{2} \Gamma_{B}-\frac{\Delta m_{B}}{2}-\frac{i}{2} y \Gamma_{B} \\
\mu_{H}=m_{H}-\frac{i}{2} \Gamma_{H}=m_{B}+\frac{\Delta m_{B}}{2}-\frac{i}{2}\left(\Gamma_{B}-\frac{\Delta \Gamma_{B}}{2}\right)=m_{B}-\frac{i}{2} \Gamma_{B}+\frac{\Delta m_{B}}{2}+\frac{i}{2} y \Gamma_{B}
\end{gathered}
$$

From PDG98 [10, $x \equiv \frac{\Delta m_{B}}{\Gamma_{B}} \sim 0.7$, while $y \equiv \frac{\Delta \Gamma_{B}}{2 \Gamma_{B}} \leq 10^{-2}$ is theoretically expected [1].

The mixing parameter $p_{i}, q_{i}$ are related by 12

$$
\frac{q_{1}}{p_{1}}=\operatorname{tg} \frac{\theta}{2} e^{i \phi}, \quad \frac{q_{2}}{p_{2}}=\operatorname{ctg} \frac{\theta}{2} e^{i \phi}
$$

where $\theta$ and $\phi$ are complex phases in general. For real $\theta$ and $\phi, 0<\theta<\pi, 0 \leq \phi<2 \pi$.

From Eq.(1), The mass difference between $B^{0}$ and $\bar{B}^{0}$ is:

$$
M_{B^{0}}-M_{\bar{B}^{0}}=\left(\mu_{L}-\mu_{H}\right) \frac{p_{1} q_{2}-p_{2} q_{1}}{p_{1} q_{2}+p_{2} q_{1}}=\left(\mu_{L}-\mu_{H}\right) \cos \theta
$$

where $\underset{B^{0}}{M_{(-)}}=m_{B^{0}}-\frac{i}{2} \Gamma_{B^{0}}$.

Using the Bell-Steinberg unitarity relation [13,

$$
\left|<B_{H}\right| B_{L}>\mid \leq \frac{\Gamma_{L} \Gamma_{H}}{\left|\mu_{L}^{*}-\mu_{H}\right|}=\frac{\sqrt{1-y^{2}} \Gamma_{B}}{|i-x| \Gamma_{B}} \simeq 0.8
$$

This constraint is more relaxed than that in $K^{0}-\bar{K}^{0}$ complex where $\left|<K_{L}\right| K_{S}>\mid \leq 0.06$. So, $\mid B_{H}>$ and $\mid B_{L}>$ are likely unorthogonal. The more relaxed constraint of Eq.(5) perhaps indicates the large $\mathrm{CP}$ or $\mathrm{CPT}$ violation in $B^{0}$ system. 
The initially $\mid B^{0}>$ or $\mid \bar{B}^{0}>$ will evolve after a proper time $t$ to

$$
\begin{aligned}
\mid B^{0}(t)> & =g_{+}(t)\left|B^{0}>+\bar{g}_{+}(t)\right| \bar{B}^{0}> \\
\mid \bar{B}^{0}(t)> & =g_{-}(t)\left|\bar{B}^{0}>+\bar{g}_{-}(t)\right| B^{0}>
\end{aligned}
$$

where

$$
\begin{array}{r}
g_{+}(t)=f_{+}(t)+\cos \theta f_{-}(t), \quad g_{-}(t)=f_{+}(t)-\cos \theta f_{-}(t) \\
\bar{g}_{+}(t)=\sin \theta e^{i \phi} f_{-}(t), \quad \bar{g}_{-}(t)=\sin \theta e^{-i \phi} f_{-}(t)
\end{array}
$$

and

$$
\begin{aligned}
& f_{+}(t)=\frac{1}{2}\left(e^{-i \mu_{L} t}+e^{-i \mu_{H} t}\right)=e^{-i m_{B} t-\frac{1}{2} \Gamma_{B} t} \operatorname{ch}\left(\frac{i x-y}{2} \Gamma_{B} t\right) \\
& f_{-}(t)=\frac{1}{2}\left(e^{-i \mu_{L} t}-e^{-i \mu_{H} t}\right)=e^{-i m_{B} t-\frac{1}{2} \Gamma_{B} t} \operatorname{sh}\left(\frac{i x-y}{2} \Gamma_{B} t\right)
\end{aligned}
$$

The probability for $\mid B^{0}>$ in a proper time $t$ to transform into $\mid B^{0}>$ is:

$$
P_{B^{0}(t) \rightarrow B^{0}}=\left|<B^{0}\right| B^{0}(t)>\left.\right|^{2}=\left|g_{+}(t)\right|^{2}
$$

Similarly, we can define $P_{B^{0}(t) \rightarrow \bar{B}^{0}}, P_{\bar{B}^{0}(t) \rightarrow B^{0}}$, and $P_{\bar{B}^{0}(t) \rightarrow \bar{B}^{0}}$.

So, the mixing-induced CPT and T asymmetries can be defined as

$$
A_{C P T}(t) \equiv \frac{P_{B^{0}(t) \rightarrow B^{0}}-P_{\bar{B}^{0}(t) \rightarrow \bar{B}^{0}}}{P_{B^{0}(t) \rightarrow B^{0}}+P_{\bar{B}^{0}(t) \rightarrow \bar{B}^{0}}}=\frac{2 \operatorname{Re}\left[\cos \theta \operatorname{sh}\left(\frac{i x-y}{2} \Gamma_{B} t\right)\left(\operatorname{ch}\left(\frac{i x-y}{2} \Gamma_{B} t\right)\right)^{*}\right]}{\left|\operatorname{ch}\left(\frac{i x-y}{2} \Gamma_{B} t\right)\right|^{2}+|\cos \theta|^{2}\left|\operatorname{sh}\left(\frac{i x-y}{2} \Gamma_{B} t\right)\right|^{2}},
$$

and

$$
A_{T}(t) \equiv \frac{P_{B^{0}(t) \rightarrow \bar{B}^{0}}-P_{\bar{B}^{0}(t) \rightarrow B^{0}}}{P_{B^{0}(t) \rightarrow \bar{B}^{0}}+P_{\bar{B}^{0}(t) \rightarrow B^{0}}}=\frac{\left|e^{i \phi}\right|^{2}-\left|e^{-i \phi}\right|^{2}}{\left|e^{i \phi}\right|^{2}+\left|e^{-i \phi}\right|^{2}}
$$

Some analysis can lead to the following conclusions [14]:

(1) CPT invariance requires $\cos \theta=0$, and thus $\theta=\frac{\pi}{2}$.

(2) $\mathrm{T}$ invariance requires $\phi=0$;

(3) CP conservation requires $\cos \theta=0$ ( and thus $\left.\theta=\frac{\pi}{2}\right)$ and $\phi=0$.

In Standard Model, CPT is conserved and $\left|\frac{q}{p}\right|-1=\left|e^{i \phi}\right|-1=\frac{1}{2} \operatorname{Im} \frac{\Gamma_{12}}{M_{12}} \sim \mathcal{O}\left(10^{-3}\right)$ [21]. Thus, the direct $\mathrm{T}$ violating asymmetry is about the order of $\mathcal{O}\left(10^{-3}\right)$. From the experience in $\mathrm{K}$ system, we guess that the CPT violating effects may be smaller than the CP violating effects. 
Under the above assumption, it is convenient to introduce a complex $\theta^{\prime}$ and two real $\phi_{0}, \phi^{\prime}$ by

$$
\begin{array}{cc}
\theta=\frac{\pi}{2}+\theta^{\prime}, & \theta^{\prime}=\operatorname{Re} \theta^{\prime}+i \operatorname{Im} \theta^{\prime} \\
\phi=\phi_{0}+i \phi^{\prime}, & \phi_{0}=\operatorname{Re} \phi, \phi^{\prime}=\operatorname{Im} \phi
\end{array}
$$

where $\operatorname{Re} \theta^{\prime}, \operatorname{Im} \theta^{\prime}, \phi_{0}, \phi^{\prime}$ are all real, and $\left|\theta^{\prime}\right|<<1, \quad\left|\phi^{\prime}\right|<<1$. The relation between $\phi_{0}$ and CKM phase $\beta$ is $\phi_{0}=-2 \beta$.

Then, we have a very simple relation

$$
\begin{aligned}
& \cos \theta=-\theta^{\prime}, \quad \sin \theta=1 \\
& e^{i \phi}=e^{i \phi_{0}}\left(1-\phi^{\prime}\right)
\end{aligned}
$$

Here we only keep terms up to the first order of $\theta^{\prime}$ and $\phi^{\prime}$.

From PDG98 [10], The mixing parameter $\frac{\Delta \Gamma_{B}}{\Gamma_{B}}$ has not been measured experimentally up to now. We further assume $y=0$ in order to simplify the formulations below. Thus,

$$
\operatorname{ch}\left(\frac{i x-y}{2} \Gamma_{B} t\right)=\cos \frac{\Delta m_{B} t}{2}, \quad \operatorname{sh}\left(\frac{i x-y}{2} \Gamma_{B} t\right)=i \sin \frac{\Delta m_{B} t}{2}
$$

From Eq.(10), Eq.(11), Eq.(13) and Eq.(14), we obtain

$$
\begin{aligned}
A_{C P T}(t) & =\frac{2 \operatorname{Im} \theta^{\prime} \sin \Delta m_{B} t}{1+\cos \Delta m_{B} t} \\
A_{T}(t) & =-2 \phi^{\prime}
\end{aligned}
$$

So, the mixing-induced CPT asymmetry is proportional to $\operatorname{Im} \theta^{\prime}$, and the mixing-induced $\mathrm{T}$ asymmetry is proportional to $\operatorname{Im} \phi=\phi^{\prime}$.

Now, we discuss two cases:

(1) Final state is not CP eigenstate

We study the decays $B^{0} \rightarrow X l^{+} \nu, \bar{B}^{0} \rightarrow \bar{X} l^{-} \nu$. From $\Delta B=\Delta Q$ rule, the decays of $B^{0} \rightarrow \bar{X} l^{-} \nu, \bar{B}^{0} \rightarrow X l^{+} \nu$ are forbidden.

For the allowed processes, we define the amplitude:

$$
<X l^{+} \nu|H| B^{0}>=A, \quad<\bar{X} l^{-} \nu|H| \bar{B}^{0}>=A^{*}
$$


So the asymmetry of semileptonic decay rates are

$$
\begin{aligned}
D_{1}(f, t) & \equiv \frac{\Gamma\left(B^{0}(t) \rightarrow X l^{+} \nu\right)-\Gamma\left(\overline{B^{0}}(t) \rightarrow \bar{X} l^{-} \nu\right)}{\Gamma\left(B^{0}(t) \rightarrow X l^{+} \nu\right)+\Gamma\left(\bar{B}^{0}(t) \rightarrow \bar{X} l^{-} \nu\right)}=\frac{P_{B^{0}(t) \rightarrow B^{0}-P_{\bar{B}^{0}(t) \rightarrow \bar{B}^{0}}}}{P_{B^{0}(t) \rightarrow B^{0}+P_{\bar{B}}^{0}(t) \rightarrow \bar{B}^{0}}}=A_{C P T}(t) \\
D_{2}(f, t) & \equiv \frac{\Gamma\left(B^{0}(t) \rightarrow \bar{X} l^{-} \nu\right)-\Gamma\left(\overline{B^{0}}(t) \rightarrow X l^{+} \nu\right)}{\Gamma\left(B^{0}(t) \rightarrow \bar{X} l^{-} \nu\right)+\Gamma\left(\overline{B^{0}}(t) \rightarrow X l^{+} \nu\right)}=\frac{P_{B^{0}(t) \rightarrow \bar{B}^{0}}-P_{\bar{B}^{0}(t) \rightarrow B^{0}}}{P_{B^{0}(t) \rightarrow \bar{B}^{0}}+P_{\overline{B^{0}}(t) \rightarrow B^{0}}}=A_{T}(t)
\end{aligned}
$$

Eq.(16) shows that CPT and T asymmetry can lead to CP asymmetry. One can use the CP asymmetry of semileptonic B decays to measure the CPT and T violation parameter.

(2) Final state is CP eigenstate

The decay rate for an initial $B^{0}$ or $\bar{B}^{0}$ transform into a CP eigenstate $f$ is

$$
\begin{aligned}
\Gamma\left(B^{0}(t) \rightarrow f\right)=e^{-\Gamma_{B} t}|A|^{2} & \left\{\frac{1+\cos \Delta m_{B} t}{2}+\operatorname{Im} \theta^{\prime} \sin \Delta m_{B} t+\left|\frac{\bar{A}}{A}\right|^{2}\left(1-2 \phi^{\prime}\right) \frac{1-\cos \Delta m_{B} t}{2}\right. \\
- & \left.\operatorname{Im}\left[e^{i \phi_{0}} \frac{\bar{A}}{A}\left(\sin \Delta m_{B} t-\phi^{\prime} \sin \Delta m_{B} t+i \theta^{\prime *}\left(1-\cos \Delta m_{B} t\right)\right)\right]\right\} \\
\Gamma\left(\bar{B}^{0}(t) \rightarrow f\right)=e^{-\Gamma_{B} t}|A|^{2}\{ & \left(1+2 \phi^{\prime}\right) \frac{1-\cos \Delta m_{B} t}{2}+\left|\frac{\bar{A}}{A}\right|^{2}\left(\frac{1+\cos \Delta m_{B} t}{2}-\operatorname{Im} \theta^{\prime} \sin \Delta m_{B} t\right) \\
+ & \left.\operatorname{Im}\left[e^{i \phi_{0}} \frac{\bar{A}}{A}\left(\sin \Delta m_{B} t+\phi^{\prime} \sin \Delta m_{B} t+i \theta^{\prime}\left(1-\cos \Delta m_{B} t\right)\right)\right]\right\}
\end{aligned}
$$

where $A \equiv A\left(B^{0} \rightarrow f\right)$ and $\bar{A} \equiv A\left(\bar{B}^{0} \rightarrow f\right)$.

For $f=J / \psi K_{S}, \frac{\bar{A}}{A}=-1$, the CP asymmetry is

$$
\begin{aligned}
D\left(J / \psi K_{S}, t\right)= & \frac{\Gamma\left(B^{0}(t) \rightarrow J / \psi K_{S}\right)-\Gamma\left(\overline{B^{0}}(t) \rightarrow J / \psi K_{S}\right)}{\Gamma\left(B^{0}(t) \rightarrow J / \psi K_{S}\right)+\Gamma\left(\bar{B}^{0}(t) \rightarrow J / \psi K_{S}\right)} \\
= & \sin \phi_{0} \sin \Delta m_{B} t+R e \theta^{\prime} \cos \phi_{0}\left(1-\cos \Delta m_{B} t\right)+\operatorname{Im} \theta^{\prime} \sin \Delta m_{B} t \\
& -\operatorname{Im} \theta^{\prime} \sin \phi_{0}\left(1-\cos \Delta m_{B} t\right)-\phi^{\prime}\left(1-\cos \Delta m_{B} t\right)+\phi^{\prime} \sin \phi_{0} \sin \Delta m_{B} t
\end{aligned}
$$

For $f=J / \psi K_{L}, \frac{\bar{A}}{A}=+1$, the $\mathrm{CP}$ asymmetry is

$$
\begin{aligned}
D\left(J / \psi K_{L}, t\right)= & \frac{\Gamma\left(B^{0}(t) \rightarrow J / \psi K_{L}\right)-\Gamma\left(\overline{B^{0}}(t) \rightarrow J / \psi K_{L}\right)}{\Gamma\left(B^{0}(t) \rightarrow J / \psi K_{L}\right)+\Gamma\left(\bar{B}^{0}(t) \rightarrow J / \psi K_{L}\right)} \\
= & -\sin \phi_{0} \sin \Delta m_{B} t-R e \theta^{\prime} \cos \phi_{0}\left(1-\cos \Delta m_{B} t\right)+I m \theta^{\prime} \sin \Delta m_{B} t \\
& +\operatorname{Im} \theta^{\prime} \sin \phi_{0}\left(1-\cos \Delta m_{B} t\right)-\phi^{\prime}\left(1-\cos \Delta m_{B} t\right)-\phi^{\prime} \sin \phi_{0} \sin \Delta m_{B} t
\end{aligned}
$$

\section{Cascade decays}

We have discussed the $B^{0}-\bar{B}^{0}$ mixing in the previous section. Now we turn to the cascade mixing involving both neutral B and neutral K systems in succession. Neglecting CPT violating 
effects in the neutral K system, the weak eigenstates of the neutral $\mathrm{K}$ mesons can be represented by the usual form:

$$
\begin{aligned}
\mid K_{S}> & =\frac{1}{\sqrt{\left|p_{K}^{2}\right|+\left|q_{K}\right|^{2}}}\left[p_{K}\left|B^{0}>+q_{K}\right| \bar{B}^{0}>\right] \\
\mid K_{L}> & =\frac{1}{\sqrt{\left|p_{K}^{2}\right|+\left|q_{K}\right|^{2}}}\left[p_{K}\left|B^{0}>-q_{K}\right| \bar{B}^{0}>\right]
\end{aligned}
$$

and their eigenvalues are

$$
\mu_{S(L)}=m_{K} \stackrel{(+)}{-\Delta m_{K}}-i \frac{\Gamma_{S(L)}}{2}
$$

where $m_{K}$ is the average of the $K_{S}$ and $K_{L}$ masses, $\Gamma_{S, L}$ are the $K_{S, L}$ widths.

The time evolution of the neutral $\mathrm{K}$ mesons can be easily obtained

$$
\begin{aligned}
\mid K^{0}(t)> & =e_{+}(t)\left|K^{0}>+\frac{q_{K}}{p_{K}} e_{-}(t)\right| \bar{K}^{0}> \\
\mid \bar{K}^{0}(t) & >=\frac{p_{K}}{q_{K}} e_{-}(t)\left|K^{0}>+e_{+}(t)\right| \bar{K}^{0}>
\end{aligned}
$$

where

$$
e_{ \pm}(t)=\frac{1}{2}\left(e^{-i \mu_{S}} \pm e^{-i \mu_{L}}\right)
$$

Consider the decay chain $B \rightarrow J / \psi+K \rightarrow J / \psi+[f]$ where $f$ can be $2 \pi, 3 \pi$ and $\pi l \nu$ as shown in Fig.1. Other decay modes of the neutral K mesons are neglected because of either very small fractions or less physical interest for this paper. We first give the formulations of the most complicated case where the final state $f=\pi l \nu$.

According to [15], the decay amplitude of the cascade decay $B^{0} \stackrel{t_{1}}{\rightarrow} J / \psi+K \stackrel{t_{2}}{\rightarrow} J / \psi+\left[\pi^{-} l^{+} \nu\right]$ is

$$
\begin{gathered}
A\left(B^{0} \stackrel{t_{1}}{\rightarrow} J / \psi+K \stackrel{t_{2}}{\rightarrow} J / \psi+\left[\pi^{-} l^{+} \nu\right]\right)=g_{+}\left(t_{1}\right) A\left(B^{0} \rightarrow J / \psi K^{0}\right) e_{+}\left(t_{2}\right) A\left(K^{0} \rightarrow \pi^{-} l^{+} \nu\right) \\
+\bar{g}_{+}\left(t_{1}\right) A\left(\bar{B}^{0} \rightarrow J / \psi \bar{K}^{0}\right) \frac{p_{K}}{q_{K}} e_{-}\left(t_{2}\right) A\left(K^{0} \rightarrow \pi^{-} l^{+} \nu\right)
\end{gathered}
$$

We assume that the transition amplitude for $\mathrm{B}$ and $\mathrm{K}$ decays satisfy the $\Delta S=\Delta Q$ rule and $\mathrm{CP}, \mathrm{CPT}$ invariance. There is no experimental signal of the violation $\Delta S=\Delta Q$ rule. The assumption of $\mathrm{CP}$ conservation in the transition amplitude for $\mathrm{B}$ and $\mathrm{K}$ decays is valid because 
$\frac{A\left(\bar{B}^{0} \rightarrow J / \psi \bar{K}^{0}\right)}{A\left(B^{0} \rightarrow J / \psi K^{0}\right)}=-1$ to a very high degree and the direct CP violation in $K^{0}-\bar{K}^{0}$ system is very small $\left(\operatorname{Re}\left(\epsilon^{\prime} / \epsilon\right) \sim 10^{-3}\right)$. We further neglect the small $\mathrm{CP}$ violations in $K^{0}-\bar{K}^{0}$ mixing, thus $\frac{q_{K}}{p_{K}}=1$.

Under the above assumptions, the decay rate of the cascade decay $B^{0} \stackrel{t_{1}}{\rightarrow} J / \psi+K \stackrel{t_{2}}{\rightarrow} J / \psi+$ $\left[\pi^{\mp} l^{ \pm} \nu\right]$ is

$$
\begin{aligned}
& \Gamma\left(B^{0}, J / \psi+\left[\pi^{\mp} l^{ \pm} \nu\right]\right) \equiv \Gamma\left(B^{0} \stackrel{t_{1}}{\rightarrow} J / \psi+K \stackrel{t_{2}}{\rightarrow} J / \psi+\left[\pi^{\mp} l^{ \pm} \nu\right]\right) \\
& \propto e^{-\Gamma_{B} t_{1}}\left\{e ^ { - \Gamma _ { S } t _ { 2 } } \left[1+\sin \phi_{0} \sin \Delta m_{B} t_{1}+R e \theta^{\prime} \cos \phi_{0}\left(1-\cos \Delta m_{B} t_{1}\right)+\operatorname{Im} \theta^{\prime} \sin \Delta m_{B} t_{1}\right.\right. \\
& \left.+I m \theta^{\prime} \sin \phi_{0}\left(1-\cos \Delta m_{B} t_{1}\right)-\phi^{\prime}\left(1-\cos \Delta m_{B} t_{1}\right)-\phi^{\prime} \sin \phi_{0} \sin \Delta m_{B} t_{1}\right] \\
& +e^{-\Gamma_{L} t_{2}}\left[1-\sin \phi_{0} \sin \Delta m_{B} t_{1}-\operatorname{Re} \theta^{\prime} \cos \phi_{0}\left(1-\cos \Delta m_{B} t_{1}\right)+I m \theta^{\prime} \sin \Delta m_{B} t_{1}\right. \\
& \left.-I m \theta^{\prime} \sin \phi_{0}\left(1-\cos \Delta m_{B} t_{1}\right)-\phi^{\prime}\left(1-\cos \Delta m_{B} t_{1}\right)+\phi^{\prime} \sin \phi_{0} \sin \Delta m_{B} t_{1}\right] \\
& \pm 2 e^{-\frac{1}{2}\left(\Gamma_{S}+\Gamma_{L}\right) t_{2}}\left[\cos \Delta m_{B} t_{1} \cos \Delta m_{K} t_{2}+\cos \phi_{0} \sin \Delta m_{B} t_{1} \sin \Delta m_{K} t_{2}\right. \\
& -R e \theta^{\prime} \sin \phi_{0}\left(1-\cos \Delta m_{B} t_{1}\right)+\operatorname{Im} \theta^{\prime} \sin \Delta m_{B} t_{1} \cos \Delta m_{K} t_{2} \\
& +I m \theta^{\prime} \cos \phi_{0}\left(1-\cos \Delta m_{B} t_{1}\right) \sin \Delta m_{K} t_{2}+\phi^{\prime}\left(1-\cos \Delta m_{B} t_{1}\right) \cos \Delta m_{K} t_{2} \\
& \left.\left.-\phi^{\prime} \cos \phi_{0} \sin \Delta m_{B} t_{1} \sin \Delta m_{K} t_{2}\right]\right\}
\end{aligned}
$$

Similarly, the decay rate of the cascade decay $\bar{B}^{0} \stackrel{t_{1}}{\rightarrow} J / \psi+K \stackrel{t_{2}}{\rightarrow} J / \psi+\left[\pi^{ \pm} l^{\mp} \nu\right]$ is

$$
\begin{aligned}
& \Gamma\left(\bar{B}^{0}, J / \psi+\left[\pi^{ \pm} l^{\mp} \nu\right]\right) \equiv \Gamma\left(\bar{B}^{0} \stackrel{t_{1}}{\rightarrow} J / \psi+K \stackrel{t_{2}}{\rightarrow} J / \psi+\left[\pi^{ \pm} l^{\mp} \nu\right]\right) \\
& =\Gamma\left(B^{0} \stackrel{t_{1}}{\rightarrow} J / \psi+K \stackrel{t_{2}}{\rightarrow} J / \psi+\left[\pi^{\mp} l^{ \pm} \nu\right]\right)\left(\theta^{\prime} \rightarrow-\theta^{\prime}, \phi_{0} \rightarrow-\phi_{0}, \phi^{\prime} \rightarrow-\phi^{\prime}\right)
\end{aligned}
$$

Because we have neglected the small CP-violating effects in K system, only $K_{S} \rightarrow 2 \pi$ and $K_{L} \rightarrow 3 \pi$ are possible. The decay rate for the cascade decays of the $f=2 \pi$ and $f=3 \pi$ are:

$$
\begin{aligned}
& \left.\quad \stackrel{(-)}{B^{0}}, J / \psi+[2 \pi]\right) \equiv \Gamma\left(B^{0} \stackrel{(-)}{\rightarrow} J / \psi+K_{S} \stackrel{t_{2}}{\rightarrow} J / \psi+[2 \pi]\right) \\
& \propto 4 e^{-\Gamma_{B} t_{1}}\left\{e ^ { - \Gamma _ { S } t _ { 2 } } \left[1 \stackrel{(-)}{+} \sin \phi_{0} \sin \Delta m_{B} t_{1} \stackrel{(-)}{+} \operatorname{Re} \theta^{\prime} \cos \phi_{0}\left(1-\cos \Delta m_{B} t_{1}\right)^{+}+\operatorname{Im} \theta^{\prime} \sin \Delta m_{B} t_{1}\right.\right. \\
& \left.\left.\quad+\operatorname{Im} \theta^{\prime} \sin \phi_{0}\left(1-\cos \Delta m_{B} t_{1}\right)^{(+)}-\phi^{\prime}\left(1-\cos \Delta m_{B} t_{1}\right)-\phi^{\prime} \sin \phi_{0} \sin \Delta m_{B} t_{1}\right]\right\}
\end{aligned}
$$

and

$$
\left.\left.\Gamma \stackrel{(-)}{\left(B^{0}\right.}, J / \psi+[3 \pi]\right) \equiv \Gamma \stackrel{(-)}{B^{0} \stackrel{t_{1}}{\rightarrow}} J / \psi+K_{L} \stackrel{t_{2}}{\rightarrow} J / \psi+[3 \pi]\right)
$$




$$
\begin{gathered}
\propto 4 e^{-\Gamma_{B} t_{1}}\left\{e ^ { - \Gamma _ { S } t _ { 2 } } \left[1 \stackrel{(+)}{-} \sin \phi_{0} \sin \Delta m_{B} t_{1} \stackrel{(+)}{-} \operatorname{Re} \theta^{\prime} \cos \phi_{0}\left(1-\cos \Delta m_{B} t_{1}\right) \stackrel{(-)}{+} \operatorname{Im} \theta^{\prime} \sin \Delta m_{B} t_{1}\right.\right. \\
\left.\left.+\operatorname{Im} \theta^{\prime} \sin \phi_{0}\left(1-\cos \Delta m_{B} t_{1}\right)^{(+)}-\phi^{\prime}\left(1-\cos \Delta m_{B} t_{1}\right)+\phi^{\prime} \sin \phi_{0} \sin \Delta m_{B} t_{1}\right]\right\}
\end{gathered}
$$

\section{The determination of the parameter $\theta$ and $\phi$}

1. $\phi^{\prime}$

From Eq.(16) and [5],

$$
A_{T}(t)=\frac{\Gamma\left(B^{0}(t) \rightarrow \bar{X} l^{-} \nu\right)-\Gamma\left(\bar{B}^{0}(t) \rightarrow X l^{+} \nu\right)}{\Gamma\left(B^{0}(t) \rightarrow \bar{X} l^{-} \nu\right)+\Gamma\left(\bar{B}^{0}(t) \rightarrow X l^{+} \nu\right)}=\frac{N^{++}-N^{--}}{N^{++}+N^{--}}=-2 \phi^{\prime}
$$

where $N^{++}, N^{--}$are the same-sign dilepton events.

$\phi^{\prime}$ can be measured by the semileptonic decays of the B mesons or by the same-sign dilepton ratios suggested in [5].

\section{2. $\operatorname{Im} \theta^{\prime}$}

From Eq.(16) and [5],

$$
A_{C P T}(t)=\frac{\Gamma\left(B^{0}(t) \rightarrow X l^{+} \nu\right)-\Gamma\left(\overline{B^{0}}(t) \rightarrow \bar{X} l^{-} \nu\right)}{\Gamma\left(B^{0}(t) \rightarrow X l^{+} \nu\right)+\Gamma\left(\bar{B}^{0}(t) \rightarrow \bar{X} l^{-} \nu\right)}=\frac{N^{+-}-N^{-+}}{N^{+-}+N^{-+}}=\frac{2 \operatorname{Im} \theta^{\prime} \sin \Delta m_{B} t}{1+\cos \Delta m_{B} t}
$$

where $N^{+-}, N^{-+}$are opposite-sign dilepton events.

Thus, $\operatorname{Im} \theta^{\prime}$ can be measured by the semileptonic decays of the B mesons or by the oppositesign dilepton ratios. For the dileptonic decays in Eq.(29) and Eq.(30), they correspond to the case of $C=-1$ where $C$ is the charge conjugation number of the $B^{0} \bar{B}^{0}$ pair.

Another method is: from Eq.(27) and Eq.(28),

$$
\begin{aligned}
& \frac{\Gamma\left(B^{0}, J / \psi+[2 \pi]\right)-\Gamma\left(\bar{B}^{0}, J / \psi+[2 \pi]\right)}{\Gamma\left(B^{0}, J / \psi+[2 \pi]\right)+\Gamma\left(\bar{B}^{0}, J / \psi+[2 \pi]\right)}+\frac{\Gamma\left(B^{0}, J / \psi+[3 \pi]\right)-\Gamma\left(\overline{\left.B^{0}, J / \psi+[3 \pi]\right)}\right.}{\Gamma\left(B^{0}, J / \psi+[3 \pi]\right)+\Gamma\left(\bar{B}^{0}, J / \psi+[3 \pi]\right)} \\
& =2\left[\operatorname{Im} \theta^{\prime} \sin \Delta m_{B} t_{1}-\phi^{\prime}\left(1-\cos \Delta m_{B} t_{1}\right)\right]
\end{aligned}
$$

Using the known $\phi^{\prime}$ value from the semileptonic decays or the dilepton ratios, $\operatorname{Im} \theta^{\prime}$ can be determined by Eq.(31). But this method is not good for experiment because their branching ratios are smaller than the semileptonic decays.

3. $\sin \phi_{0}$ and the absolute value of the $\cos \phi_{0}$ and $\operatorname{Re} \theta^{\prime}$ 
From Eq.(27) and (28), we can obtain the time-dependent asymmetry of the decay rates

$$
\begin{aligned}
& \frac{\left[\Gamma\left(B^{0}, J / \psi+[2 \pi]\right)-\Gamma\left(\bar{B}^{0}, J / \psi+[2 \pi]\right)\right]}{\left[\Gamma\left(B^{0}, J / \psi+[2 \pi]\right)+\Gamma\left(\bar{B}^{0}, J / \psi+[2 \pi]\right)\right]}-\frac{\left[\Gamma\left(B^{0}, J / \psi+[3 \pi]\right)-\Gamma\left(\bar{B}^{0}, J / \psi+[3 \pi]\right)\right]}{\left[\Gamma\left(B^{0}, J / \psi+[3 \pi]\right)+\Gamma\left(\bar{B}^{0}, J / \psi+[3 \pi]\right)\right]} \\
& =2\left[\sin \phi_{0} \sin \Delta m_{B} t_{1}+\operatorname{Re}^{\prime} \cos \phi_{0}\left(1-\cos \Delta m_{B} t_{1}\right)\right]
\end{aligned}
$$

The asymmetry of Eq.(32) is twice as much as the usual CP asymmetry in the decay of $B \rightarrow J / \psi K_{S}$ because we have used the decay mode of $B \rightarrow J / \psi K_{L}$ to double the asymmetry. We will discuss the problem caused by the detection of $K_{L}$ later.

There are two contributions in the asymmetry of Eq.(32). The $\sin \Delta m_{B} t_{1}$ term is an odd function of time while the $\left(1-\cos \Delta m_{B} t_{1}\right)$ term is an even function. These two terms can be distinguished experimentally by measuring the decay time order of $B^{0}$ abd $\bar{B}^{0}$ decays. The detail of this method is given in [16]. Here we only use this method to distinguish the $\sin \Delta m_{B} t_{1}$ and $\left(1-\cos \Delta m_{B} t_{1}\right)$ terms.

Like [16], define two asymmetries

$$
a_{-}(f, t)=\frac{(\bar{\Gamma}+\tilde{\Gamma})-(\Gamma+\tilde{\bar{\Gamma}})}{(\bar{\Gamma}+\tilde{\Gamma})+(\Gamma+\widetilde{\bar{\Gamma}})}, \quad a_{+}(f, t)=\frac{(\bar{\Gamma}+\tilde{\bar{\Gamma}})-(\Gamma+\bar{\Gamma})}{(\bar{\Gamma}+\widetilde{\bar{\Gamma}})+(\Gamma+\bar{\Gamma})}
$$

where $\Gamma, \bar{\Gamma}, \widetilde{\Gamma}$ and $\widetilde{\bar{\Gamma}}$ are defined in [16, and the subscript $(-)$ or $(+)$ denotes an odd or even function of time. The measurement of asymmetry $a_{-}(f, t)$ requires measuring the decay time order.

Thus the two terms of Eq.(32) can be distinguished by

$$
A_{-}\left(t_{1}\right)=a_{-}\left(f_{1}, t_{1}\right)-a_{-}\left(f_{2}, t_{1}\right)=2 \sin \phi_{0} \sin \Delta m_{B} t_{1}
$$

and

$$
A_{+}\left(t_{1}\right)=a_{+}\left(f_{1}, t_{1}\right)-a_{+}\left(f_{2}, t_{1}\right)=2 \operatorname{Re} \theta^{\prime} \cos \phi_{0}\left(1-\cos \Delta m_{B} t_{1}\right)
$$

where $f_{1}$ and $f_{2}$ represent the final states $J / \psi+[2 \pi]$ and $J / \psi+[3 \pi]$.

In Eq.(34), the asymmetry is often used to measure the direct $\mathrm{CP}$ violation when $\mathrm{CPT}$ invariance holds. The direct $\mathrm{CP}$ violation in $B \rightarrow J / \psi K$ decays is at the order of $\mathcal{O}\left(10^{-3}\right)$. So the measurement of the CPT violation in Eq.(34) can only reach the accuracy up to $10^{-2}$ 
because of the dilution of direct $\mathrm{CP}$ violation in $B \rightarrow J / \psi K$ decay and the $\mathrm{CP}$ violation in $K^{0}-\bar{K}^{0}$ mixing.

From the Eq.(33) and Eq.(34), the values of $\sin \phi_{0}$ and $R e \theta^{\prime} \cos \phi_{0}$ can be obtained. So $\cos \phi_{0}$ and $R e \theta^{\prime}$ can be determined except for the ambiguity of their sign. This ambiguity can be solved by the cascade decays where $f=\pi l \nu$.

4. The sign of $\cos \phi_{0}$ and $R e \theta^{\prime}$

From Eq.(25) and Eq.(26),

$$
\begin{aligned}
& \frac{\left[\Gamma\left(B^{0}, J / \psi+\left[\pi^{-} l^{+} \nu\right]\right)-\Gamma\left(B^{0}, J / \psi+\left[\pi^{+} l^{-} \nu\right]\right)+\Gamma\left(\overline{B^{0}}, J / \psi+\left[\pi^{-} l^{+} \nu\right]\right)-\Gamma\left(\overline{B^{0}}, J / \psi+\left[\pi^{+} l^{-} \nu\right]\right)\right.}{\left[\Gamma\left(B^{0}, J / \psi+\left[\pi^{-} l^{+} \nu\right]\right)+\Gamma\left(B^{0}, J / \psi+\left[\pi^{+} l^{-} \nu\right]\right)+\Gamma\left(\bar{B}^{0}, J / \psi+\left[\pi^{-} l^{+} \nu\right]\right)+\Gamma\left(\bar{B}^{0}, J / \psi+\left[\pi^{+} l^{-} \nu\right]\right)\right.} \\
& =\frac{-2 e^{-\frac{1}{2}\left(\Gamma_{S}+\Gamma_{L}\right) t_{2}} \operatorname{Re} \theta^{\prime} \sin \phi_{0}\left(1-\cos \Delta m_{B} t_{1}\right)}{\left\{e^{-\Gamma_{S} t_{2}}\left[1+I m \theta^{\prime} \sin \phi_{0}\left(1-\cos \Delta m_{B} t_{1}\right)-\phi^{\prime} \sin \phi_{0} \sin \Delta m_{B} t_{1}\right]\right.} \\
& \left.\quad+e^{-\Gamma_{L} t_{2}}\left[1-I m \theta^{\prime} \sin \phi_{0}\left(1-\cos \Delta m_{B} t_{1}\right)+\phi^{\prime} \sin \phi_{0} \sin \Delta m_{B} t_{1}\right]\right\} \\
& \sim \frac{-2 e^{-\frac{1}{2}\left(\Gamma_{S}+\Gamma_{L}\right) t_{2}} \operatorname{Re}^{\prime} \sin \phi_{0}\left(1-\cos \Delta m_{B} t_{1}\right)}{e^{-\Gamma_{S} t_{2}}+e^{-\Gamma_{L} t_{2}}}
\end{aligned}
$$

and

$$
\begin{aligned}
& \frac{\left[\Gamma\left(B^{0}, J / \psi+\left[\pi^{-} l^{+} \nu\right]\right)-\Gamma\left(B^{0}, J / \psi+\left[\pi^{+} l^{-} \nu\right]\right)-\Gamma\left(\overline{B^{0}}, J / \psi+\left[\pi^{-} l^{+} \nu\right]\right)+\Gamma\left(\overline{B^{0}}, J / \psi+\left[\pi^{+} l^{-} \nu\right]\right)\right.}{\left[\Gamma\left(B^{0}, J / \psi+\left[\pi^{-} l^{+} \nu\right]\right)+\Gamma\left(B^{0}, J / \psi+\left[\pi^{+} l^{-} \nu\right]\right)+\Gamma\left(\bar{B}^{0}, J / \psi+\left[\pi^{-} l^{+} \nu\right]\right)+\Gamma\left(\overline{B^{0}}, J / \psi+\left[\pi^{+} l^{-} \nu\right]\right)\right.} \\
& =\frac{A}{B} \sim \frac{2 e^{-\frac{1}{2}\left(\Gamma_{S}+\Gamma_{L}\right) t_{2}}\left[\cos \Delta m_{B} t_{1} \cos \Delta m_{K} t_{2}+\cos \phi_{0} \sin \Delta m_{K} t_{2} \sin \Delta m_{B} t_{1}\right]}{e^{-\Gamma_{S} t_{2}}+e^{-\Gamma_{L} t_{2}}}
\end{aligned}
$$

where

$$
\begin{aligned}
A= & 2 e^{-\frac{1}{2}\left(\Gamma_{S}+\Gamma_{L}\right) t_{2}}\left[\cos \Delta m_{B} t_{1} \cos \Delta m_{K} t_{2}+\cos \phi_{0} \sin \Delta m_{K} t_{2}\left(\sin \Delta m_{B} t_{1}\right.\right. \\
& \left.-\operatorname{Im} \theta^{\prime}\left(1-\cos \Delta m_{B} t_{1}\right)+\phi^{\prime} \sin \Delta m_{B} t_{1}\right) \\
& \left.-\operatorname{Im} \theta^{\prime} \sin \Delta m_{B} t_{1} \cos \Delta m_{K} t_{2}-\phi^{\prime}\left(1-\cos \Delta m_{B} t_{1}\right)\right] \\
B= & e^{-\Gamma_{S} t_{2}}\left[1+\operatorname{Im} \theta^{\prime} \sin \phi_{0}\left(1-\cos \Delta m_{B} t_{1}\right)-\phi^{\prime} \sin \phi_{0} \sin \Delta m_{B} t_{1}\right] \\
& +e^{-\Gamma_{L} t_{2}}\left[1-\operatorname{Im} \theta^{\prime} \sin \phi_{0}\left(1-\cos \Delta m_{B} t_{1}\right)+\phi^{\prime} \sin \phi_{0} \sin \Delta m_{B} t_{1}\right]
\end{aligned}
$$

From Eq.(35) and Eq.(36), the sign of $\cos \phi_{0}$ and $\operatorname{Re} \theta^{\prime}$ can be measured. Actually, we do not need to use the Eq.(35) since we have known the value of $\operatorname{Re} \theta^{\prime} \cos \phi_{0}$. 


\section{Feasibility and discussions}

In order to meet the goal of a three standard deviation measurement for the $B^{0}-\bar{B}^{0}$ mixing parameter $\theta$ and $\phi$, the relation between the number of $B^{0} \bar{B}^{0}$ pairs and the asymmetry is [17]:

$$
N_{B^{0} \overline{B^{0}}}=\frac{1}{B \epsilon_{r} \epsilon_{t}[(1-2 W) d \cdot \delta A]^{2}}
$$

where $\delta A=\frac{A}{3} ; A$ is the asymmetry of the decay ratios; $B$ is the branching ratio of the decay; $\epsilon_{r}$ is the reconstruction efficiency of the final state $f ; \epsilon_{t}$ is the tagging efficiency; $W$ is the fraction of incorrect tags; $d$ is the dilution factor which takes into account the loss in asymmetry due to fitting, time integration, and/or the mixing of the tagged decay.

Sometimes, another relation is used:

$$
N_{e f f}=\frac{1}{[(1-2 W) d \cdot \delta A]^{2}}
$$

where $N_{\text {eff }}$ is the effective number of the decay events.

The B-factories can accumulate $1.8 \times 10^{8} B \bar{B}$ pairs every year [19], and the effective event number of Eq.(38) for $B \rightarrow J / \psi K_{S}$ is taken to be $2.7 \times 10^{5}$ in LHC-B[20]. Table 1 and Table 2 give some parameters [17] and the minimum asymmetries (lower bound) which can be achieved in B-factory and LHC-B.

Now we discuss how to determine the two complex phase $\theta$ and $\phi$.

(1) $\phi^{\prime}$ and $\operatorname{Im} \theta^{\prime}$ can be measured in semileptonic decays and dileptonic decays given in Eq.(29) and Eq.(30). Semileptonic decays have larger branching ratio but smaller detection efficiency, while dileptonic decays have larger detection efficiency but smaller branching ratio than semileptonic decays. In B-factory (with $1.8 \times 10^{8} B^{0} \bar{B}^{0}$ pairs per year), the $\phi^{\prime}$ and $\operatorname{Im} \theta^{\prime}$ can be measured to an accuracy of $2 \times 10^{-3}$ for semileptonic decays and $7 \times 10^{-3}$ for dileptonic decays at $3 \sigma$ level. In LHC-B (with $4 \times 10^{12} b \bar{b}$ pairs every snow mass year), the $\phi^{\prime}$ and $\operatorname{Im} \theta^{\prime}$ can be measured to an accuracy of $10^{-5}$ for semileptonic decays and $4 \times 10^{-5}$ for dileptonic decays with $3 \sigma$ standard deviation.

(2) $R e \theta^{\prime} \cos \phi_{0}$ can be determined clearly from Eq.(34). In Eq.(32) the decay of $K_{L} \rightarrow 3 \pi$ is used in order to increase the asymmetry factor and reduce the ambiguity or error caused by 
Table 1. Branching Ratios and Reconstruction efficiencies for the cascade decays and semileptonic decays.

\begin{tabular}{|c|c|c|}
\hline \hline Decay mode & Braching Ratio $B$ & Reconstruction effeciency $\epsilon_{r}$ \\
\hline$B \rightarrow J / \psi K_{S} \rightarrow J / \psi+[2 \pi]$ & $5 \times 10^{-4}$ & 0.61 \\
\hline$B \rightarrow J / \psi K_{L} \rightarrow J / \psi+[3 \pi]$ & $5 \times 10^{-4} \times \frac{1}{3}$ & 0.4 \\
\hline$B \rightarrow J / \psi K_{S} \rightarrow J / \psi+[\pi l \nu]$ & $5 \times 10^{-4} \cdot 1.2 \times 10^{-3}$ & 0.61 \\
\hline$B \rightarrow J / \psi K_{L} \rightarrow J / \psi+[\pi l \nu]$ & $5 \times 10^{-4} \times \frac{2}{3}$ & 0.4 \\
\hline$B \rightarrow l \nu+X$ & 0.1 & 1 \\
\hline$J / \psi \rightarrow l^{+} l^{-}$ & 0.14 & - \\
\hline
\end{tabular}

Tag efficiencies and Asymmetry dilution at B-factory and LHC-B

\begin{tabular}{|c|c|c|}
\hline \hline & at B-factory & at LHC-B \\
\hline Tag efficiency $\epsilon_{t}$ & 0.48 & 0.61 \\
\hline Asymmetry dilution $d$ & $0.61\left(\right.$ for $\left.B_{d}\right)$ & 0.61 \\
\hline
\end{tabular}

the unknown $\phi^{\prime}$ and $\operatorname{Im} \theta^{\prime}$. The $K_{L}$ detection is a challenge for experiment. In [18], one idea to catch $K_{L}$ by using Fe sampling after the electromagnetic calorimeter is suggested. In B-factory, the accuracy of measuring $R e \theta^{\prime} \cos \phi_{0}$ can reach 0.07 . So, it is likely that $R e \theta^{\prime} \cos \phi_{0}$ can not be measured in B-factory. In LHC-B, the accuracy of measuring $R e \theta^{\prime} \cos \phi_{0}$ can reach 0.01 . We have no confidence that $R e \theta^{\prime} \cos \phi_{0}$ can be measured clearly with $3 \sigma$ standard deviation in LHC-B. If it can be measured, $R e \theta^{\prime}$ must be larger than 0.01 . This will be the largest CPT violation effects.

(3) $\sin \phi_{0}$ is usually suggested to be measured in $B \rightarrow J / \psi K_{S}\left(\rightarrow \pi^{+} \pi^{-}\right)$which gives the $A(t)=\sin \phi_{0} \sin \Delta m_{B} t$ in Standard Model. If the CPT violating effects are considered, the asymmetry is modified to Eq.(18). In order to cancel the errors caused by $\phi^{\prime}$ and $\operatorname{Im} \theta^{\prime}$, we use 
Table 2. Comparison between the Minimum of Asymmetries with $3 \sigma$ standard deviation at B-factory and LHC-B

\begin{tabular}{|c|c|c|}
\hline \hline \multirow{2}{*}{ Decay mode } & \multicolumn{2}{|c|}{ Asymmetry $A$} \\
\cline { 2 - 3 } & at B-factory & at LHC-B \\
\hline$B \rightarrow J / \psi K_{S} \rightarrow J / \psi+[2 \pi]$ & 0.08 & $1.2 \times 10^{-2}$ \\
\hline$B \rightarrow J / \psi K_{L} \rightarrow J / \psi+[3 \pi]$ & 0.17 & $2.6 \times 10^{-2}$ \\
\hline$B \rightarrow J / \psi K_{S} \rightarrow J / \psi+[\pi l \nu]$ & 1.44 & 0.27 \\
\hline$B \rightarrow J / \psi K_{L} \rightarrow J / \psi+[\pi l \nu]$ & 0.06 & 0.01 \\
\hline$B \rightarrow l \nu+X$ & $1.7 \times 10^{-3}$ & $1 \times 10^{-5}$ \\
\hline$B \bar{B} \rightarrow l^{+} l^{-}$ & $7 \times 10^{-3}$ & $4 \times 10^{-5}$ \\
\hline
\end{tabular}

the Eq.(33) to determine $\sin \phi_{0}$.

(4) $\sin \phi_{0}$ can be measured in B-factory and LHC-B as discussed above, but the value of $\phi_{0}$ has two ambiguity. If $\operatorname{Re} \theta^{\prime} \cos \phi_{0}$ can be measured, the only ambiguity is the sign of $\cos \phi_{0}$. This can be solved by measuring the ratios of the cascade decays $B \rightarrow J / \psi+K \rightarrow J / \psi+[\pi l \nu]$ given in Eq.(36). Because the very small branching ratio of decay $B \rightarrow J / \psi+K \rightarrow J / \psi+[\pi l \nu]$ in $t_{2} \leq 2 \tau_{S}$, the determination of the sign of $\cos \phi_{0}$ can only be done in LHC-B. Table 2 gives the lower bound of measuring for asymmetry in cascade decay is 0.2 . This is possible because $\left|\cos \phi_{0}\right|>0.5$, we have taken $0.3 \leq \sin \phi_{0} \leq 0.88$ [21] for our estimation.

In conclusion, the cascade decays provide an elegant and beautiful place to study the CPT violation caused by the $B^{0}-\bar{B}^{0}$ mixing.

\section{Acknowledgment}

This work is supported in part by National Natural Science Foundation of China and the Grant of State Commission of Science and Technology of China. 


\section{References}

[1] CPLEAR Collaboration (A. Angelopoulos et al.) Phys.Lett.B444(1998) 43-51.

[2] Fermilab KTeV collaboration, seminar talk by P.Shawhan.

[3] CPLEAR Collaboration (A. Angelopoulos et al.) Phys.Lett.B444(1998) 52-60.

[4] V.A. Kostelecký, Phys.Rev.Lett80(1998) 1818.

[5] M. Kobayashi, A.I. Sanda, Phys.Rev.Lett69(1992) 3139.

[6] Z.Z. Xing, Phys.Rev.D50 (1994) R2957; Phys.Lett.B450 (1999) 202.

[7] Y.I. Azimov, Phys.Rev.D42 (1990) 3705.

[8] B. Kayser, hep-ph/9709382.

[9] C.C. Meca, J.P. Silva, Phys.Rev.Lett81(1998) 1377.

[10] PDG98, Eur.Phys.J.C3 (1998)

[11] Y. Nir, H.R. Quinn, Annu.Rev.Nucl.Part.Sci.42 (1992) 211.

[12] T.D. Lee, C.S. Wu, Annu.Rev.Nucl.Sci16(1966) 471.

[13] CURRENT PHYSICS SOURCES AND COMMENTS Vol.5 CP Violation, edited by L.Wolfenstein.

[14] M.Hayakawa, and A.I.sanda, DPNU 93-05.

[15] B. Kayser, L. Stodolsky, Phys.Lett.B359(1995) 343.

[16] M. Gronau, Phys.Rev.Lett63(1989) 1451.

[17] SLAC-353, LBL-27856, CALT-1588, October, 1992,

[18] SLAC-400, 529, November, 1992, B factories.

[19] The BaBar Collaboration, Technical Design Report SLAC-R-95-457, March, 1995. 
[20] T. Nakada, hep-ex/9502005.

[21] Y.Nir, hep-ph/9709301, or in the Proceeding of the 18th International Symposium on Lepton Photon Interactions, Hamberg, Germany, July 28-August 11997. 

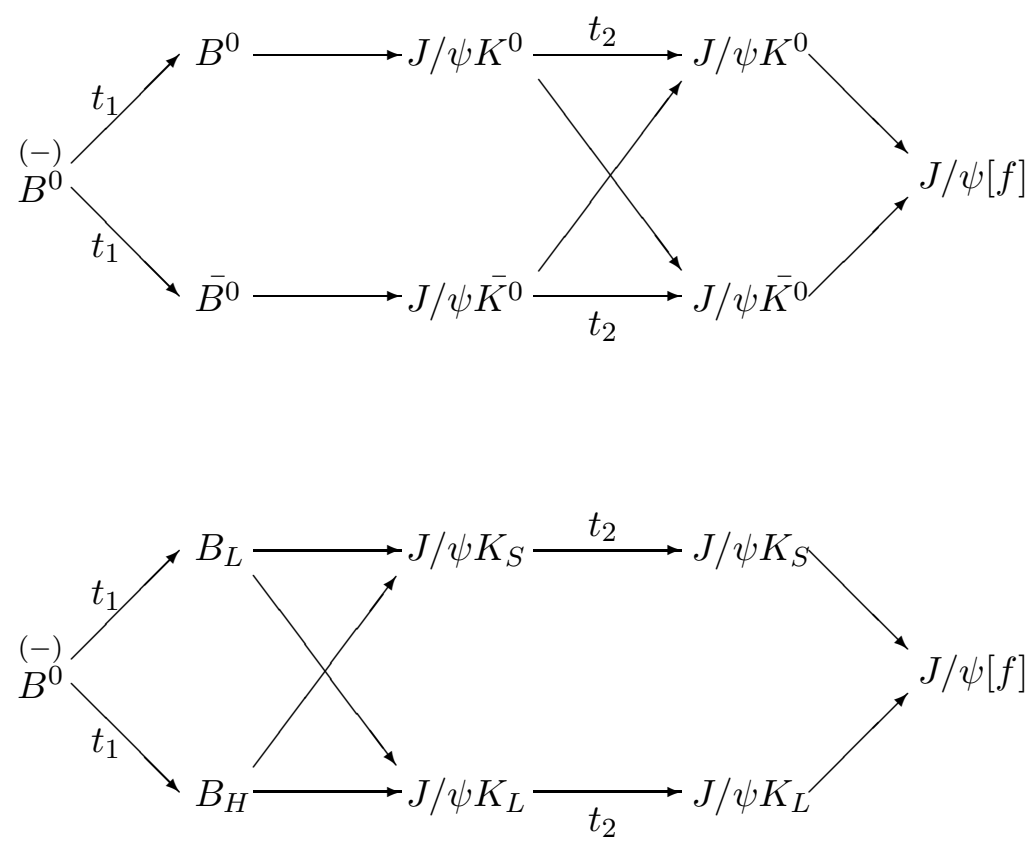

Fig.1 The cascade decay chains $B \rightarrow J / \psi K \rightarrow J / \psi[f]$ 